\title{
Pathophysiology of Cerebral Edema-A Comprehensive Review
}

\author{
Tara Dalby ${ }^{1} \quad$ Elyana Wohl ${ }^{2} \quad$ Michael Dinsmore ${ }^{1}$ \\ Lakshmikumar Venkatraghavan' \\ ${ }^{1}$ Department of Anesthesia and Pain Medicine, Toronto Western \\ Hospital, University Health Network, University of Toronto, \\ Ontario, Canada \\ 2Department of Anesthesia, Notre-Dame Hospital, Montreal, \\ Quebec, Canada
}

Zoe Unger $^{1} \quad$ Tumul Chowdhury ${ }^{1}$

\begin{abstract}
Address for correspondence Lashmi Venkatraghavan, MD, FRCA, FRCPC, Department of Anesthesia and Pain Medicine, Toronto Western Hospital, University Health Network, University of Toronto, University of Toronto 399, Bathurst Street, Toronto Ontario, M5T 2S8, Canada (e-mail: lashmi.venkatraghavan@uhn.ca).
\end{abstract}

J Neuroanaesthesiol Crit Care 2021;8:163-172.

\begin{abstract}
Keywords

- cerebral edema

- blood-brain barrier

- aquaporins

Cerebral edema is a condition where an excess of cerebral water accumulates due to primary neurological or non-neurological causes. Cerebral edema complicates many brain pathologies causing additional injury often in excess of the original neurological insult. Classic descriptions divide cerebral edema into cytotoxic, vasogenic, interstitial, and osmotic subtypes. The interplay of different mechanisms is important in the clinical manifestations. Recent research has advanced our understanding of the molecular pathophysiology of cerebral edema, exposing the central role of aquaporins and specific ion channels. The aim of this review is to provide a comprehensive overview of the molecular pathophysiology of cerebral edema including unique disease specific mechanisms.
\end{abstract}

\section{Introduction}

The fluid compartments in the brain and the barriers between them differ significantly from the systemic compartments and are unique to the central nervous system (CNS). Cerebral edema is a condition of excess of fluid in the brain resulting from either neurological or non-neurological causes. Cerebral edema can result from a variety of mechanisms. These include cellular, vasogenic, interstitial, and osmotic mechanisms. Causes of cerebral edema are widespread and can be divided into neurological and non-neurological categories. Neurological causes include ischemia, hemorrhage, hypoxia, trauma, tumors, infections, and hydrocephalus. Common non-neurologic causes include acute hypertension, liver failure, metabolic derangements, and high-altitude cerebral edema (HACE). Cerebral edema affects all age groups, genders, and ethnic groups. Incidence of cerebral edema varies among the individual clinical conditions and the actual frequency is often under-reported due to nonspecific symptoms.

Cerebral edema resulting from various causes have unique patterns based on the interplay of different mechanisms.

published online

December 3, 2020
DOI https://doi.org/ 10.1055/s-0040-1721165 ISSN 2348-0548.
In addition, in some cases, a combination of these mechanisms can also occur. To understand the different mechanisms for the formation of cerebral edema is crucial for the management of specific types of cerebral edema as well as future developments in disease specific management. Therefore, this review aims to provide a comprehensive overview on the pathophysiology of cerebral edema. In addition, this review also highlights the mechanism of cerebral edema in a few common conditions including focal or global ischemia, intracranial hemorrhage, traumatic brain injury (TBI), liver failure, and high-altitude sickness.

\section{Background}

The brain composition has unique anatomical and physiological characteristics.

\section{Fluid Compartments in the Brain}

The cerebral fluid compartments have different composition, regulation, and interactions as compared with other body fluids. In contrast to three fluid compartments (intracellular (c) 2020. Indian Society of Neuroanaesthesiology and Critical Care. This is an open access article published by Thieme under the terms of the Creative Commons Attribution-NonDerivative-NonCommercial-License, permitting copying and reproduction so long as the original work is given appropriate credit. Contents may not be used for commercial purposes, or adapted, remixed, transformed or built upon. (https://creativecommons.org/licenses/by-nc-nd/4.0/)

Thieme Medical and Scientific Publishers Pvt. Ltd., A-12, 2nd Floor, Sector 2, Noida-201301 UP, India 
[ICF], interstitial [ISF], and intravascular) in the systemic circulation, there is an additional cerebrospinal fluid (CSF) compartment in the cerebral circulation ( - Fig. 1A, B). Among these compartments, the ICF is the largest one accounting for $\sim 70 \%$ of the volume, ${ }^{1}$ followed by the CSF and intravascular compartments. ISF compartment has negligible volume and it is in continuity with the CSF compartment. The composition and volume of the ICF rely on energy-dependent processes. The $\mathrm{Na}^{+}-\mathrm{K}^{+}$ATPase is crucial for maintaining the transmembrane electrochemical gradient that drives other transporters, and thus maintains the cell volume. Cerebral edema occurs when there is expansion of either the ICF and/ or ISF, caused by either dysfunction of ion transporter or the membrane barrier. The overall adverse outcomes (morbidity and mortality) of cerebral edema are due to a combination of various mechanisms, including disruption of neuronal transmission, electrolyte derangement, cell death, and ischemia from cerebral compartment syndrome.

\section{Anatomy of the Brain Barrier Systems}

The fluid compartments in the brain are normally tightly separated from the systemic circulation by physical and chemical barriers. This sustains a constant internal environment optimal for neuronal function. The barriers include the blood-brain barrier (BBB), the blood CSF barrier, and the outer brain barrier (OBB) (-Fig. 2). ${ }^{2}$ The integrity of the BBB, which isolates the brain parenchyma from systemic vascular circulation, is the key factor implicated in the development of cerebral edema. The BCSFB and OBB isolate the CSF from the systemic circulation and

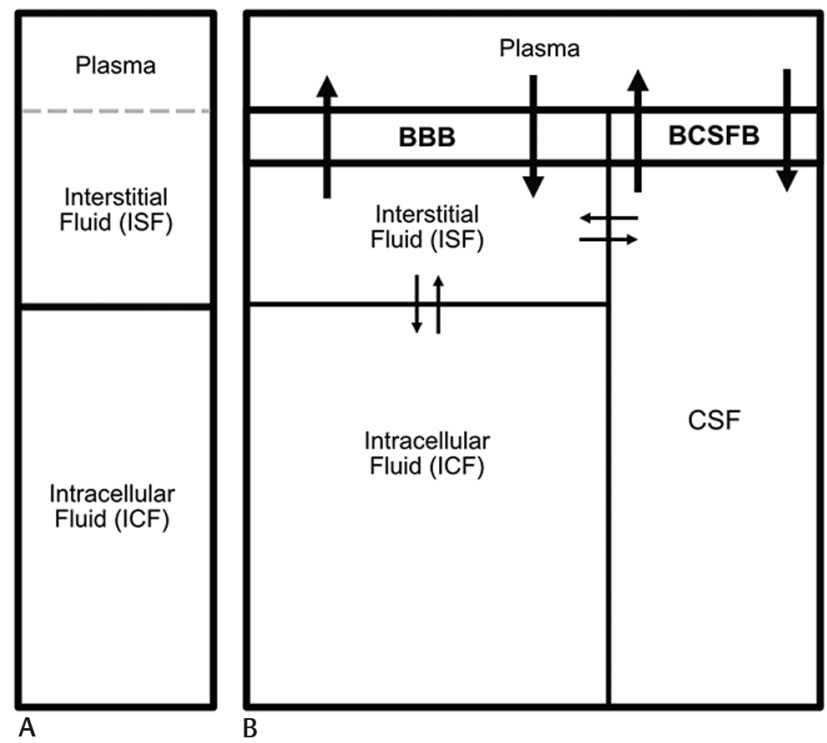

Fig. 1 Systemic and cerebral fluid compartments. Systemic body fluids are separated into 3 compartments $(\mathbf{A})$ The majority of water in the body is located in the intracellular fluid compartment (ICF) and the rest is in the extracellular compartment (ECF). The ECF comprises the interstitial fluid (ISF) and the plasma. The brain has 4 fluid compartments, with the additional compartment of the cerebrospinal fluid (CSF) (B) The interstitial and CSF compartments freely exchange with one another, not separated by any barrier. The plasma, on the other hand, is tightly separated from the interstitial compartment and the CSF by the blood-brain barrier (BBB) and blood CSF barrier (BSCFB), respectively.

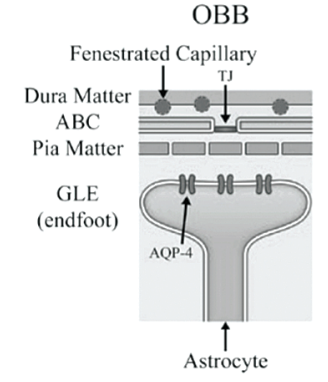

BBB
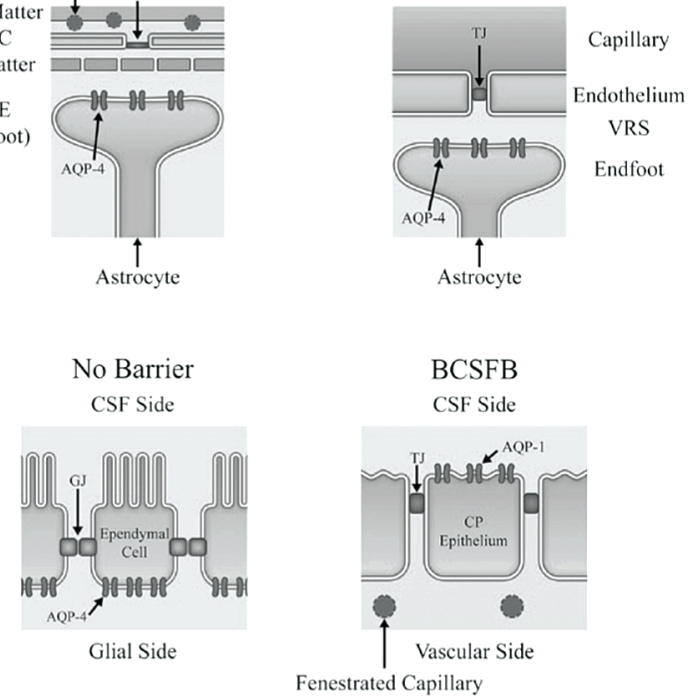

Fig. 2 Fluid compartment barriers within the central nervous system (CNS). The brain barriers isolate the fluid compartments from each other and the systemic circulation. The outer brain barrier (OBB) separates the interstitial fluid from the systemic circulation mainly through the arachnoid barrier cells (ABC), which contain tight junctions (TJ). The blood-brain barrier (BBB) isolates the vascular compartment from the CNS with TJ between endothelial cells. Aquaporin-4 (APQ4) is expressed on the astrocyte foot processes adjacent to the $\mathrm{BBB}$ and is involved in water transport intracellularly but not across the BBB. The blood cerebrospinal fluid (CSF) barrier (BCSFC) is formed by TJ between choroid plexus (CP) endothelium. (Reproduced with permission from Nakada T, Kwee IL. Fluid dynamics inside the brain barrier: current concept of interstitial flow, glymphatic flow, and cerebrospinal fluid circulation in the brain. Neuroscientist. 2019; 25:155-166.)

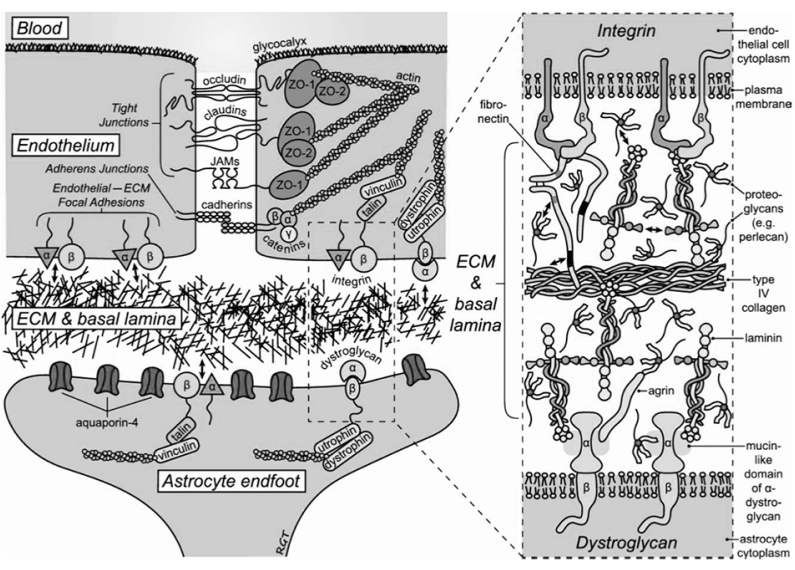

Fig. 3 The blood-brain barrier (BBB). The cerebral endothelium contains tight junctions and has no fenestrations. This restricts paracellular diffusion of water and other molecules from the systemic circulation into the brain. Tight junction proteins such as occludin, claudins, cadherins, zona occludens 1 (ZO-1) and 2 (ZO-2), and junctional adhesion molecules (JAMs) interact with the cytoskeletal components to regulate paracellular diffusion. The glycocalyx provides the first barrier to diffusion, coating the lumen of the endothelium. The final barrier is the extracellular matrix (ECM) and basal lamina, containing type IV collagen. The astrocytes line the BBB with foot processes, expressing aquaporin 4 , which permits fluid flux intracellularly. (Reproduced with permission from Abbott NJ, Pizzo ME, Preston JE, et al. The role of brain barriers in fluid movement in the CNS: is there a "glymphatic" system? Acta Neuropathologica. 2018; 135:387-407.) 
are formed mainly by the epithelial tight junctions of the choroid plexus, and arachnoid barrier cells, respectively ( Fig. 2). ${ }^{2}$ The barrier function of the BBB is accomplished by a series of resistors to molecular diffusion, which interact dynamically to regulate the internal environment within the CNS ( - Fig. 3 ).

Further to anatomical details, the endothelial surface layer, comprising circulating plasma proteins and the glycocalyx, is the first component of the BBB on the vascular luminal side ( - Fig. 3 ). ${ }^{3}$ The glycocalyx is a gel-like coating on the endothelial lumen that relies on a constant supply of blood flow for maintenance and regulation, hence blood supply is critical for maintaining a fully functional BBB. ${ }^{3}$ The discovery of the glycocalyx has modified our understanding of the Starling forces that drives the diffusion across vascular endothelial walls in vivo. The subglycocalyx has a much lower oncotic pressure than the plasma, and when substituted into the Starling equation, the expected flow with an intact glycocalyx is far less. ${ }^{4}$ Interestingly, the cerebral glycocalyx is denser in the brain than in other tissues such as the heart or lungs, ${ }^{5}$ suggesting a critical barrier function in the CNS. However, it is also incredibly fragile and can be damaged by ischemia, reperfusion, hyperglycemia, sepsis, inflammation, and shock. ${ }^{6}$ Hence, the glycocalyx is implicated in the pathogenesis of cerebral edema of various etiologies. ${ }^{6}$

Fluid flux from the vascular lumen occurs primarily across the interendothelial cell junctions of the capillaries. ${ }^{3}$ The capillary endothelial cells are a major component of the physical barrier due to lack of fenestrations and presence of tight junctions and adherent

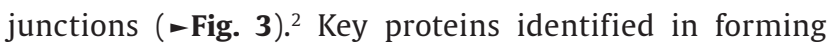
these junctions include claudins, occludins and junctional adhesion molecules. ${ }^{7}$ The continuous rather than fenestrated cerebral capillary basement membrane provides yet another barrier fluids must cross to enter the brain. ${ }^{2}$ Thus, the endothelial cells provide the major physical barrier in maintaining CNS integrity.

Astrocytes are involved in forming the CNS side of the BBB and additionally provide a lymphatic-like clearance mechanism within the brain (-Fig. 3). ${ }^{1}$ Each astrocyte extends at least one foot-process to contact and envelop a capillary surface. ${ }^{1}$ These end-feet contain transport channels regulating the entry of nutrients into the CNS. ${ }^{5}$ The water channel aquaporin 4 (APQ4) is densely expressed on the end-feet, effectively lining the BBB and BCSFB..$^{2,5.8}$ APQ4 has a critical roles in water regulation, nutrient sensing, and delivery in the brain. ${ }^{2,8}$ The movement of fluid through the brain via astrocytes and specifically the APQ4 has been described as lymphatic-like, or a "glymphatic" system. ${ }^{1}$ This system is essential in not only the formation but also the clearance of cerebral edema.

\section{Intracranial Compliance}

Cerebral edema can lead to a cerebral compartment syndrome. The intact skull is nonexpandable; therefore, as per the Monro-Kellie doctrine, the net volume of its contents, blood, CSF, and brain tissue, remains constant. ${ }^{9}$ In cerebral edema as the volume of brain parenchyma increases, CSF translocation and a reduction in intracranial blood volume occur to compensate. If the increase is rapid, these compensatory mechanisms may be overwhelmed, leading to cerebral ischemia from both reduced blood flow and a sudden increase in intracranial pressure (ICP). It starts a vicious cycle of malignant ICP that can lead to herniation: the compression of vital brain stem structures through the foramen magnum and ultimately death.

\section{Clearance of Fluids from the CNS}

The mechanism of fluid clearance from the CNS remains incompletely understood. Fluid clearance is important for protecting against the development of cerebral edema and in promoting its resolution. Excess water may leave the CNS either via bulk flow down a pressure gradient perivascularly,,$^{10}$ or via the astral-glial, or glymphatic system. ${ }^{1}$ One of the important routes for removal of excess ISF from the brain is via fluid tracks along the basement membrane in the walls of capillaries to the cervical lymph nodes..$^{10}$ The glymphatic system refers to a dynamic lymphatic-like network of APQ4 expressing astrocytes involved in fluid clearance (-Fig. 4). The glymphatic flow has diurnal variations, with flow highest during sleep. ${ }^{11}$ APQ4 knockout studies have demonstrated differences in the involvement of APQ4 in the edema formation and clearance between the brain and the spinal cord. ${ }^{12}$ In animal models of cerebral edema caused by inflammation, upregulation of APQ4 is associated with worse edema in the acute phase; however, upregulation also promotes the resolution of edema in the subacute phase by increased clearance and inhibition of microglial activation. ${ }^{13}$ Clearance of fluid through the glymphatic system may yet prove an important modifiable factor in cerebral edema management.

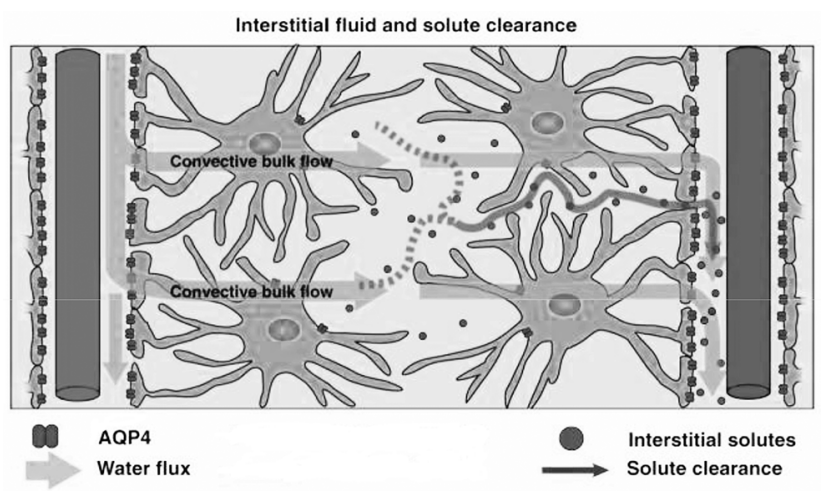

Fig. 4 "Glymphatic" flow of fluid in the brain. Aquaporins are present on the end-feet of astrocytes lining the cerebral capillaries. Water flows from the interstitial space into astrocytes down an osmotic gradient. Water continues to follow the movement of ions within the astrocyte, eventually exiting the intercellular space back into the interstitial space via aquaporins. Excess fluid is eliminated from the brain via a paravenous route, tracking along the basement membrane along the capillary wall eventually draining to the cervical lymph nodes. (Reproduced with permission from Lliff JJ, Wang M, Liao $Y$ et al. A paravascular pathway facilitates CSF flow through the brain parenchyma and the clearance of interstitial solutes, including amyloid B. Sci Transl Med. 2012; 4:147.) 


\section{Molecular Mechanisms of Cerebral Edema}

Accumulation of excess fluid in the brain may arise from dysfunction of the cell membrane (cytotoxic edema), damage to the BBB (vasogenic edema), outflow of CSF from intraventricular space to the interstitial space (interstitial edema), and due to water being pulled from the plasma by the brain cells due to osmotic derangements (osmotic edema) (-Table 1) ${ }^{1,14,15}$ Among these mechanisms, cytotoxic and vasogenic processes are the main pathological forces driving common brain pathologies. These mechanisms tend to co-exist due to the symbiotic dependence of an intact BBB with adequate cerebral perfusion and a healthy astro-glial system.

\section{Cytotoxic Cerebral Edema}

Cytotoxic edema develops when an osmotic gradient results in water movement from the ISF to the ICF, particularly into the astrocytes. ${ }^{16}$ An osmotic gradient is established in the context of an intact BBB from the accumulation of ions within the ICF, due to ion transporter dysfunction, which is characteristic of cerebral ischemia. Failure of the adenosine triphosphate (ATP) dependent $\mathrm{Na}^{+}-\mathrm{K}^{+}$ATPase is a common endpoint of many acute CNS pathologies. The $\mathrm{Na}^{+}-\mathrm{K}^{+}$ATPase is critical for maintaining the transmembrane electrochemical gradient; its subsequent failure leads to membrane depolarization..$^{17}$ Following depolarization, excitatory neurotransmitters are released, causing $\mathrm{Na}^{+}$and $\mathrm{Ca}^{2+}$ influx through ligand gated channels with water following. This leads to volume overload of the cells. Activation of second messenger cascades following $\mathrm{Ca}^{2+}$ influx eventually leads to apoptosis. ${ }^{17}$ Furthermore, $\mathrm{Na}^{+}$continues to enter the CNS (across the endothelial cell) into the ISF by cation channels, with $\mathrm{Cl}^{-}$and water following their electrochemical and osmotic gradients, respectively. ${ }^{18}$ The $\mathrm{Na}^{+}-\mathrm{K}+-\mathrm{Cl}^{-}$cotransporter- 1 (NKCC1) of astrocytes and endothelium is one such important cation channel. NKCC1 is upregulated in acute brain injury and a key molecular target for therapy through the inhibitor bumetanide. ${ }^{14,15,19}$ Influx of ions and hence water through this process leads to a net increase in tissue mass despite an intact BBB. The astrocytes, which express APQ4, preferentially swell in cytotoxic edema thus implicating APQ4 in water influx in the acute phase of cytotoxic edema. ${ }^{20,21}$ In addition, the monovalent cation channel sulfonylurea receptor 1 transient receptor potential melastatin 4 (SUR1-TRPM4) is involved in cytotoxic edema formation. ${ }^{22}$ The TRMP4 pore-forming unit is activated by high intracellular calcium or very low ATP, with pore sensitivity to calcium modulated by transmembrane receptor SUR1. ${ }^{22}$ SUR1-TRPM4 normally aids in calcium homeostasis by allowing cation influx when intracellular calcium is high, thus reducing the electrical gradient for further calcium influx. However, under conditions of very low intracellular ATP such as ischemic injury, the channel is activated leading to cell swelling, membrane blebbing, and eventual rupture. ${ }^{23,24}$ SUR1-TRPM4 is normally not present in healthy neuronal or vascular tissue but it is upregulated following injury from trauma or ischemia. ${ }^{22}$ Further, it is associated with the membrane $\mathrm{APQ} 4$, linking the ion and water influx in pathological conditions..$^{25}$ Thus, cytotoxic edema is primarily due to failure of ion homeostasis, leading to fluid shifts from the ISF to the ICF.

\section{Vasogenic Cerebral Edema}

Vasogenic edema is the result of BBB disruption causing the extravasation of serum proteins into the interstitial

Table 1 Classification of cerebral edema

\begin{tabular}{|c|c|c|c|c|}
\hline & Cytotoxic & Vasogenic & $\begin{array}{l}\text { Interstitial } \\
\text { (hydrocephalic) }\end{array}$ & Osmotic \\
\hline Primary mechanism & Metabolic failure & BBB breakdown & $\begin{array}{l}\text { CSF accumulation and over- } \\
\text { flow into extracellular space }\end{array}$ & Osmotic derangement \\
\hline Pathophysiology & $\begin{array}{l}\text { Failure of the } \mathrm{Na}^{+}- \\
\mathrm{K}^{+} \text {ATPase } \rightarrow \text { influx } \\
\text { of ions, (particularly } \\
\mathrm{Na}^{+} \text {) \& water } \rightarrow \\
\text { expansion of } \\
\text { ICF and volume } \\
\text { overload occurs in } \\
\text { all cells (neurons, } \\
\text { glia, endothelial } \\
\text { cells) }\end{array}$ & $\begin{array}{l}\text { BBB integrity is } \\
\text { disturbed } \rightarrow \\
\text { increased capillary } \\
\text { permeability allowing } \\
\text { fluid, an ultrafiltrate of } \\
\text { plasma, to accumulate } \\
\text { in the ISF }\end{array}$ & $\begin{array}{l}\text { Volume of CSF increases } \\
\text { due to imbalance of pro- } \\
\text { duction and clearance } \rightarrow \\
\text { increased intraventricular } \\
\text { pressure } \rightarrow \text { transependymal } \\
\text { flow of CSF periventricular } \\
\text { extracellular space }\end{array}$ & $\begin{array}{l}\text { Relatively lower plasma } \\
\text { osmolarity to intracerebral } \\
\text { osmolarity creates an } \\
\text { osmotic gradient } \rightarrow \text { water } \\
\text { diffusion across BBB } \\
\text { into ISF }\end{array}$ \\
\hline Etiology & $\begin{array}{l}\text { Anoxia, ischemia, } \\
\text { trauma, liver failure }\end{array}$ & $\begin{array}{l}\text { Tumors, Ischemia (late } \\
\text { stages), ICH, trauma, } \\
\text { infections, high-altitude } \\
\text { Cerebral edema }\end{array}$ & $\begin{array}{l}\text { Hydrocephalus, meningitis, } \\
\text { pseudotumor cerebri }\end{array}$ & $\begin{array}{l}\text { Hyponatremia, SIADH } \\
\text { and other metabolic } \\
\text { pathologies }\end{array}$ \\
\hline Contents of edema & $\begin{array}{l}\text { Water, sodium, no } \\
\text { protein }\end{array}$ & High protein fluid & CSF, low protein & Water, no protein \\
\hline Location of edema & $\begin{array}{l}\text { Within both gray } \\
\text { and white matter }\end{array}$ & $\begin{array}{l}\text { Predominantly in the } \\
\text { white matter }\end{array}$ & $\begin{array}{l}\text { Periventricularly and within } \\
\text { the white matter }\end{array}$ & Within the white matter \\
\hline BBB integrity & Intact & Breakdown & Intact & Intact \\
\hline
\end{tabular}

Abbreviations: BBB, blood-brain barrier; CSF, cerebrospinal fluid; ICF; intracellular fluid; ISF, interstitial fluid; ICH, intracerebral hemorrhage; SIADH, syndrome of inappropriate antidiuretic hormone secretion. 
space. ${ }^{14}$ Hydrostatic forces perpetuate vasogenic edema in contrast to osmotic gradients as in cytotoxic edema. Breakdown of the BBB can be due to various causes including uncontrolled hypertension, inflammation, infection, ischemia, and neoplasms. Acute CNS injury, including cytotoxic edema, causes transcriptional changes in the neurovascular unit leading to creation of "endothelial permeability pores" 14 and eventually leading to BBB breakdown. Several vascular permeability factors and membrane receptors have been identified as therapeutic targets. The endothelin-receptor $\mathrm{B}\left(\mathrm{ET}_{\mathrm{B}}-\mathrm{R}\right)$ induces a reactive state in astrocytes resulting in the upregulation of matrix-metalloproteinases (MMPs), that degrade extracellular matrix, and vascular endothelial growth factor (VEGF), which disrupts tight junction proteins on endothelial cells. ${ }^{19}$ The SUR1-TRPM4 channel is also implicated in the development of vasogenic edema. SUR1-TRPM4 is upregulated in neural and vascular tissues following CNS injury, contributing to BBB dysfunction. ${ }^{26}$ Hence, vasogenic and cytotoxic edema often occur together as one leads to the other. The interplay of the molecular mechanisms in various clinical scenarios is summarized in - Table 2. Differences in imaging between vasogenic and cytotoxic edema are shown in -Supplementary Figure S1 (available in the online version).

\section{Pathophysiology and Mechanisms of Cerebral Edema in Common Clinical Conditions Acute Ischemic Stroke}

Table 2 Mechanism of cerebral edema in various clinical scenarios

\begin{tabular}{|c|c|c|}
\hline Condition & $\begin{array}{l}\text { Type of cerebral } \\
\text { edema }\end{array}$ & Molecular mechanism and evolution \\
\hline Acute ischemic stroke & $\begin{array}{l}\text { Cytotoxic and then } \\
\text { vasogenic }\end{array}$ & $\begin{array}{l}\text { - Focal ischemia and damage to cerebral endothelium } \\
\text { - Failure of } \mathrm{Na}^{+}-\mathrm{K}^{+} \text {ATPase } \rightarrow \mathrm{Na}^{+} \text {and } \mathrm{Ca}^{2+} \text { influx through ligand gated } \\
\text { channels with water following } \\
\text { - Hypoxia inducible factor gene activation } \rightarrow \text { upregulation of SUR1-TRMP4 } \\
\text { - Vasogenic edema occurs over days to weeks caused by ischemic damage } \\
\text { to the BBB } \\
\text { - Vasogenic edema is worse if reperfusion occurs after prolonged ischemia }\end{array}$ \\
\hline $\mathrm{TBI}$ & $\begin{array}{l}\text { Vasogenic and then } \\
\text { cytotoxic }\end{array}$ & $\begin{array}{l}\text { - Mechanical shearing with primary impact-immediate diffuse BBB } \\
\text { disruption-early vasogenic edema (maximal at 4-6 hours) } \\
\text { - Early contusional edema from cell necrosis } \\
\text { - Late vasogenic edema (5 days)-inflammation due to microglial activation } \\
\text { - Cytotoxic edema (hours to days)-neuronal ischemia due to increased ICP } \\
\text { from vasogenic edema, deranged neuronal metabolism, exacerbated by } \\
\text { hypoxia and hypotension } \\
\text { - Upregulation of SUR1-TRMP4 exacerbates ion and water influx }\end{array}$ \\
\hline $\mathrm{ICH}$ & $\begin{array}{l}\text { Vasogenic and } \\
\text { cytotoxic }\end{array}$ & $\begin{array}{l}\text { - Hematoma coagulates and retracts-hydrostatic pressure draws in fluid } \\
\text { from vasculature (1-4 hours) } \\
\text { - BBB breakdown due to inflammation secondary to the presence of blood } \\
\text { clot (early vasogenic edema first } 72 \text { hours) and breakdown products (late } \\
\text { vasogenic edema-after } 72 \text { hours) } \\
\text { - Increased ICP surrounding hematoma and edema } \rightarrow \text { microvascular } \\
\text { compression } \rightarrow \text { ischemia } \rightarrow \text { cytotoxic edema }\end{array}$ \\
\hline $\mathrm{SAH}$ & $\begin{array}{l}\text { Cytotoxic and } \\
\text { vasogenic }\end{array}$ & $\begin{array}{l}\text { - Global cerebral edema not universal-associated with severe ischemic } \\
\text { injury on aneurysm rupture } \\
\text { - Widespread ischemic injury and microvascular disruption } \rightarrow \text { cytotoxic } \\
\text { edema } \\
\text { - Neuroinflammation- microglial activation and leucocyte infiltration } \rightarrow \\
\text { cytotoxic edema } \\
\text { - SUR1-TRMP4 upregulation } \\
\text { - Endothelial damage } \rightarrow \text { vasogenic edema }\end{array}$ \\
\hline Peritumoral & Vasogenic & $\begin{array}{l}\text { - } \uparrow \text { BBB permeability by substances secreted by tumor cells-mainly VEGF } \\
\text { and also by MMPs, leukotrienes, prostaglandins, nitric oxide } \\
\text { - } \quad \downarrow \text { Expression of tight junctional proteins in adjacent endothelium } \\
\text { - Mast cell activation } \rightarrow \text { inflammation } \rightarrow \text { vasogenic edema } \\
\text { - Dexamethasone suppresses VEGF production among other mechanisms } \\
\text { such as reducing cytokine and inflammatory-mediated BBB breakdown }\end{array}$ \\
\hline
\end{tabular}


Table 2 (continued)

\begin{tabular}{|c|c|c|}
\hline Condition & $\begin{array}{l}\text { Type of cerebral } \\
\text { edema }\end{array}$ & Molecular mechanism and evolution \\
\hline Fulminant liver failure & $\begin{array}{l}\text { Cytotoxic and } \\
\text { vasogenic }\end{array}$ & $\begin{array}{l}\text { - Ammonia accumulation in mitochondria of astrocytes } \rightarrow \text { oxidative stress } \\
\text { - Ammonia } \rightarrow \text { activates microglia leading to inflammation } \\
\text { - Hyponatremia worsens cerebral edema }\end{array}$ \\
\hline Global ischemia & $\begin{array}{l}\text { Cytotoxic and } \\
\text { vasogenic }\end{array}$ & $\begin{array}{l}\text { - Similar to acute ischemic stroke but more diffuse } \\
\text { - Following reperfusion-period of mismatched oxygen demand and } \\
\text { - Vulnerableads to ongoing ischemia } \\
\text { and cerebellar vermis }\end{array}$ \\
\hline $\begin{array}{l}\text { High-altitude cerebral } \\
\text { edema }\end{array}$ & Vasogenic & $\begin{array}{l}\text { - } \uparrow \text { Cerebral blood volume and BBB permeability } \\
\text { - } \uparrow \text { Cerebral blood flow in response to reduced } \mathrm{P}_{\mathrm{a}} \mathrm{O}_{2} \\
\text { - } \uparrow \text { Arterial inflow } \rightarrow \text { compression of venules } \downarrow \text { venous outflow } \\
\text { - Microbleeds in corpus collosum suggest BBB breakdown }\end{array}$ \\
\hline Infectious & $\begin{array}{l}\text { Vasogenic, cytotoxic } \\
\text { and interstitial }\end{array}$ & $\begin{array}{l}\text { - Encephalitis, meningitis, or abscess } \rightarrow \text { inflammation } \rightarrow \text { BBB breakdown } \\
\text { - Microglial activation and leucocyte infiltration } \rightarrow \text { cytotoxic edema } \\
\text { - Meningitis may } \rightarrow \text { damage to the CSF outflow pathway } \rightarrow \text { transependymal } \\
\text { extravasation of CSF }\end{array}$ \\
\hline Hydrocephalus & Interstitial & - Obstruction of CSF efflux $\rightarrow$ transependymal extravasation of CSF \\
\hline Hyponatremia & Osmotic & - Water moves down an osmotic gradient into CNS \\
\hline Radiation-induced edema & Vasogenic & $\begin{array}{l}\text { - Cell death from targeted radiation } \rightarrow \text { inflammation } \\
\text { - Reactive astrocytes express VEGF } \rightarrow \uparrow \text { BBB permeability }\end{array}$ \\
\hline Postsurgical edema & $\begin{array}{l}\text { Vasogenic and then } \\
\text { cytotoxic }\end{array}$ & $\begin{array}{l}\text { - Mechanical disruption of cells and vessels at the time of surgery } \rightarrow \\
\text { inflammatory cascade } \rightarrow \text { increased BBB permeability } \\
\text { - Perilesional hypoxia secondary to vasogenic edema } \rightarrow \text { cytotoxic edema }\end{array}$ \\
\hline
\end{tabular}

Abbreviations: BBB, blood-brain barrier; ICP, intracranial pressure; MMPs, matrix metalloproteinases; SUR1-TRMP4 sulfonylurea receptor 1 transient receptor potential melastatin 4 channel; VEGF, vascular endothelial growth factor.

Cerebral edema remains a leading cause of death in patients with massive ischemic infarcts. The amount and type of edema depend on the ischemic duration, severity, and the presence/timing of reperfusion. Deaths occurring days after stroke are often related to edema and progressive herniation. ${ }^{27}$ Cerebral edema due to ischemic stroke is primarily caused by cytotoxic edema, followed, and exacerbated by vasogenic edema. Following acute ischemia, anaerobic metabolism is inadequate to meet metabolic demands and intracellular ATP is rapidly depleted leading to failure of the $\mathrm{Na}^{+}-\mathrm{K}^{+}$ATPase leading to cytotoxic edema. ${ }^{17}$ The SUR1-TRPM4 channel is expressed in human brain specimens postinfarction and is likely a major contributor to the development of cerebral edema following focal ischemia. ${ }^{26}$ The underlying mechanism involves activation of the gene hypoxia inducible factor $1 .{ }^{24}$ Additionally, promising retrospective studies on humans and animal models of sulfonylurea drugs that inhibit the SUR1-TRPM4 appear to have better outcomes (mortality and functional independence) following ischemic stroke. ${ }^{23,24}$

The edema occurring during ischemia is initially limited by the intact BBB. Animal studies have shown that cytotoxic edema forms within 15 minutes of ischemia onset as water moves into the ICF. This is seen on diffusion-weighted magnetic resonance imaging(MRI) as reduced apparent diffusion coefficient. ${ }^{28}$ There is a linear correlation between the extent of reperfusion and the amount of edema formation, ${ }^{27}$ likely due to exacerbation of cytotoxic edema from BBB breakdown and vasogenic edema. Animal studies have shown edema is worse if reperfusion occurs after 3 hours of ischemia versus 1 hour. ${ }^{29}$ The vasogenic edema follows a different time course, with maximal fluid extravasation 3 days to 2 weeks postischemic insult. ${ }^{27}$ The mechanism of BBB damage at the level of the endothelial cell involves tight junction disruption. ${ }^{27}$ In embolic stroke, vascular damage also occurs at the site of embolism due to infarction of the vessel wall, and more distally through ischemic damage to the endothelium. ${ }^{27}$ Thus, the mechanism of edema in acute ischemic stroke is both cytotoxic and vasogenic, the degree of which is greatly influenced by the timing of reperfusion.

\section{Traumatic Brain Injury}

TBI is the result of mechanical and shearing forces in the brain, causing vascular, neuronal, and/or axonal damage. The extent of edema is the biggest predictor of outcome following TBI and accounts for up to half of the associated morbidity and mortality. ${ }^{30}$ Here, both vasogenic and cytotoxic mechanisms contribute to the formation of edema at different time points and to varying extents. Mechanical disruption of the BBB occurs immediately during and following primary impact. "Contusional edema" that develops surrounding the damaged necrotic area is from an osmotic gradient established by cellular apoptosis. ${ }^{29}$ Endothelial cells undergo cytoskeletal 
changes with degradation of tight junction and activation of inflammatory cascades following impact. ${ }^{31}$ This causes a diffuse BBB breakdown. Substance P, calcitonin G-related peptide, MMPs, and VEGF are key in producing vasogenic edema through promoting vascular permeability. ${ }^{29,32}$ The transient increased BBB permeability is maximal at 4 to 6 hours post injury. ${ }^{30}$ The second peak in BBB permeability occurs at 5 days post injury, potentially due to microglia activated by inflammatory cascades. ${ }^{31}$

Neuronal ischemia and metabolic disturbances following TBI lead to cytotoxic edema progressing from 1 hour to 7 days after the injury. ${ }^{29,30}$ As in acute ischemic stroke, vasogenic edema exacerbates the cytotoxic edema. The fluid and proteins extravasated into brain ISF and the associated increased ICP lead to the occlusion of small vessels, perpetuating further ischemic damage. ${ }^{31,33}$ SUR1-TRPM4 is upregulated in rat models of diffuse TBI, peaking at 6 hours, and its expression associated with astrocyte swelling. ${ }^{34}$

\section{Intracranial Hemorrhage}

Intracranial hemorrhage occurs from intracerebral hemorrhage (ICH), bleeding within the brain parenchyma; subarachnoid hemorrhage (SAH), bleeding into the subarachnoid space; or intraventricular hemorrhage (IVH), extension of bleeding into the ventricles mostly associated with $\mathrm{ICH}$ or SAH. ${ }^{7}$

\section{Intracerebral Hemorrhage}

ICH carries a high burden of morbidity and mortality. The initial injury causes irreversible damage to the surrounding brain parenchyma. The ensuing cerebral edema results in secondary injury of magnitude that may be far greater than the initial cause. ${ }^{35}$ Peak perihematomal edema (PHE) correlates with a poor functional outcome. ${ }^{35}$

PHE develops from a conglomeration of hydrostatic, cytotoxic, and vasogenic mechanisms with a distinctive time-course. ${ }^{36,37}$ The early ( 1 to 4 hours) phase of edema is due to perihematomal hydrostatic pressure changes. ${ }^{36,37}$ The coagulation cascade is activated causing the blood to clot and retract. Hydrostatic pressure reduces surrounding the clot, leading to fluid influx from the vasculature. ${ }^{36}$ In addition, microvascular compression from raised ICP leads to hypoperfusion that then triggers cytotoxic mechanisms. ${ }^{37}$ The initial breach in BBB and extravasation of blood products activates mast cells within seconds, leading to inflammatory cascades involving activated microglia, astrocytes and driving neutrophil and leukocyte infiltration. ${ }^{38}$ In the intermediate period (4-72 hours), thrombin activation is the major driving force behind inflammatory cascades and also directly interfering with endothelial cell interactions disrupting the BBB, perpetuating vasogenic edema. ${ }^{36}$ The inflammatory response is important in hematoma resolution in addition to exacerbating edema.

The late stage ( $>72$ hours) of PHE is due to the toxic breakdown products of the clot. ${ }^{37}$ Erythrocyte lysis occurs following complement activation, leading to the presence of free hemoglobin, a potent neurotoxin. ${ }^{39}$ Cell death occurs through inhibition of the $\mathrm{Na}^{+}-\mathrm{K}^{+}$ATPase, free radical formation, and lipid peroxidation. ${ }^{36}$ Peak PHE volume is reached between day 8 and 12 post initial injury, ${ }^{7,35}$ with the PHE causing the volume of the lesion to increase as much to $150 \%{ }^{36}$

\section{Subarachnoid Hemorrhage}

$\mathrm{SAH}$ is most often the results from the rupture of an aneurysm. Acute injury is associated with a surge in ICP, followed by secondary or delayed injury. ${ }^{40}$ Global cerebral edema occurs in a subset of patients and is an independent predictor of mortality. ${ }^{41}$ The mechanism of global cerebral edema following SAH is unclear, and an MRI study 72 hours post injury suggests a combination of vasogenic and cytotoxic mechanisms. ${ }^{41,42}$ It may have a biphasic time course, with some patients showing edema on initial presentation (6-8\%) and others developing edema later in the course of the disease (12\%). ${ }^{41}$ At the time of aneurysm rupture, ICP surges, causing a temporary arrest of cerebral perfusion causing widespread ischemic injury and leading to global edema. ${ }^{40,43,44}$ The causative factors for edema may relate to the ischemic tissue and microvascular injury during cerebral circulatory arrest. ${ }^{41}$ Delayed cerebral edema is positively associated with the use of hypertensive therapies, and is potentially caused by cerebral ischemia. ${ }^{41}$ Another causative mechanism may be "neuroinflammation," manifest as microglial activation and leucocyte infiltration leading to cytotoxic edema independent of ischemia. ${ }^{43}$ Widespread endothelial damage occurring immediately after SAH results in vasogenic edema. ${ }^{44}$ Expression of integral BBB proteins is reduced in animal models of SAH, such as endothelial tight junction proteins (occludins, claudin 5 and zona occludens- 1 [ZO-1]) maximal at 24 to 48 hours post ictus, with ZO-1 and occludins having a second reduction after 72 hours. ${ }^{7}$ MMP blockade reduces the vasogenic edema associated with $\mathrm{SAH}^{43}$ Early vasogenic edema seen on diffusion weighted MRI correlates with the Fischer grade, implicating the presence of blood products in developing vasogenic edema. ${ }^{42}$ The SUR1-TRMP4 channel is also upregulated in both human and animals following SAH. ${ }^{45}$ Inhibition of the channel with glibenclamide in a rat model of SAH leads to reduction in inflammatory markers such as tumor necrosis factor $\alpha$ (TNF- $\alpha$ ) expression, SAH-induced immunoglobulin G, and post SAH cognitive impairment. ${ }^{45}$ While APQ4 is upregulated in animals and humans following SAH, animal knockout models and blockade experiments show conflicting results regarding edema formation. ${ }^{43}$ The cerebral edema associated with SAH is a mixture of cytotoxic and vasogenic mechanisms, caused by widespread ischemic and inflammatory insults.

A major complication of SAH is delayed cerebral ischemia (DCI) occurring at 5 to 14 days after initial injury and responsible for a large amount of the associated morbidity and mortality. ${ }^{40,41}$ Etiopathogenesis of DCI may be related to increased BBB permeability, spreading cortical depolarization, loss of cerebral autoregulation, microthrombosis, and microcirculatory changes. ${ }^{46,47}$ The relationship with cerebral edema is unclear; however, early brain edema is a predictive factor for the development of DCI. ${ }^{48}$ 
Intraventricular Hemorrhage

IVH, rarely an isolated occurrence, is usually an extension of SAH or ICH into the ventricles. ${ }^{7}$ IVH may occur in the premature infant, as the periventricular germinal matrix is fragile with an immature BBB; a deficient basement membrane, underdeveloped endothelial tight junctions, and incomplete coverage of capillaries by astrocyte end feet., ${ }^{7,42}$ The main pathophysiological consequence leading to edema is hydrocephalus-accumulation of CSF due to impaired drainage. Vasogenic edema may also occur, as animal models of IVH through injection of blood or collagenase into the ventricles result in BBB disruption. ${ }^{7}$

\section{Brain Tumors}

Brain tumors (malignant gliomas, metastases, and meningiomas) are associated with cerebral edema. The amount of cerebral edema can be extensive, leading to focal neurological deficits, coma, and the patient demise. Vasogenic edema from disruption of the microvasculature appears to be the primary mechanism in peri-tumoral cerebral edema (PTE). ${ }^{49}$

Tumor cells secrete VEGF that increases BBB permeability. ${ }^{49,50}$ VEGF expression is also upregulated in brain tumors that are prone to edema. ${ }^{51}$ Notably, messenger ribonucleic acid levels of VEGF correlate with edema severity in meningioma. ${ }^{50}$ Conditions within the tumors, such as hypoxia and acidosis, can further increase VEGF expression, leading to widening of interendothelial gaps and creating fenestrations in the basement membrane..99,52 Dexamethasone suppresses VEGF production ${ }^{50}$ among other mechanisms such as reducing cytokine and inflammatory-mediated BBB breakdown. ${ }^{49}$ This may account for the unique role of steroids in cerebral edema-associated with tumors. Other factors contributing to increased BBB permeability in PTE are the leukotrienes, prostaglandins, nitric oxide MMPs, and mast cells activation..$^{53}$

The vasculature of malignant tumors has abnormal structure, contributing to vasogenic edema. Endothelium adjacent to high-grade glial tumors has reduced expression of the tight junction proteins claudins and occludins, abnormal tight junctions as well as fenestrations that are incompletely covered by an irregular basal lamina. ${ }^{51}$ Aggressiveness of the tumor correlates better with the volume of the edema than the size of the tumor itself. ${ }^{51,54}$ Dexamethasone treatment has been shown to upregulate tight junction proteins such as claudin, occludin, and ZO- 1 in vitro, ${ }^{49}$ which may be an additional mechanism for its profound effect on PTE. Aquaporins of various subtypes tend to be overexpressed in brain tumors and their adjacent vasculature, ${ }^{49}$ with a strong correlation between aquaporin expression and tumor grade. ${ }^{55}$ In addition to water balance regulation, aquaporins have been shown to facilitate cell migration, proliferation and cell adhesion, which is pertinent to tumor biology.

\section{Fulminant Liver Failure}

Cerebral edema occurs in $80 \%$ of patients with fulminant liver failure and is a leading cause of death. ${ }^{56}$ Although ammonia accumulation in the astrocytes is the most vital factor in the pathophysiology of cerebral edema in fulminant liver failure, ${ }^{56}$ other factors including damage to the BBB, inflammation, and hyponatremia also play an important role. ${ }^{57}$

Accumulation of ammonia in astrocytes follows the failure of hepatic detoxification mechanisms. Astrocytes contain the enzyme glutamine synthetase, which converts ammonia to glutamine, a precursor for the excitatory neurotransmitter glutamate. ${ }^{56}$ Accumulation of ammonia within the mitochondria leads to oxidative stress, and release of reactive oxygen species. ${ }^{58}$ Additionally, microglia in contact with ammonia release proinflammatory cytokines TNF- $\alpha$, interleukin-1, and interleukin-6 leading to activation of further microglia and establishing an inflammatory cascade, ultimately causing disruption of the BBB and promoting further cytotoxicity. ${ }^{56,57}$ The contribution of vasogenic edema in the setting of fulminant liver failure remains inconclusive, as animal models have not yet demonstrated clear evidence of BBB breakdown..$^{59}$

\section{Hypoxic-Ischemic Cerebral Edema}

Hypoxic-ischemic injury occurs following a sufficient period of asphyxia depriving the brain of oxygen. These events occur in situations such as adult cardiac arrest, or perinatally during complicated labor and delivery (e.g., umbilical cord around the neck), potentially leading to lifelong neurological deficits. The resultant cerebral edema is by both cytotoxic and vasogenic mechanisms similar to acute ischemic stroke, but occurs more diffusely. The brain is subject to ongoing ischemic injury even after flow is re-established due to mismatch in demand and delivery of oxygen. This phenomenon, called "no-reflow," may be due to disruption of microvascular control mechanisms and/or vasoconstrictive levels of extracellular $\mathrm{K}^{+}{ }^{60}$ Highly metabolically active areas such as hippocampi, thalamus, cerebral cortex, corpus striatum, and cerebellar vermis are particularly vulnerable to damage. ${ }^{60}$ Activated microglia, microvascular thrombi, and inflammatory cytokines exacerbate vasogenic edema. ${ }^{61}$ The length of circulatory arrest is the most important marker of extent and severity of cerebral damage. During fetal development, cerebral autoregulation develops between 23 and 33 weeks, placing premature infants at higher risk of ischemic damage than term infants. ${ }^{62}$

\section{High-Altitude Cerebral Edema}

HACE is a severe consequence of acute mountain sickness, caused by low partial pressure of oxygen at altitude, resulting in a hypoxic hypoxemia in un-acclimatized individuals. This may adversely affect those with reduced ability to compensate for the hypoxia induced increase in cerebral blood volume $^{63}$ The disease is rare, difficult to investigate, and the mechanisms are therefore not fully understood.

HACE appears to be vasogenically mediated through increased cerebral blood volume and BBB permeability. The vasogenic nature is supported by the extensive microhemorrhages within the corpus callosum and good response to steroids, ${ }^{64,65}$ with inflammatory pathways involving VEGF, nitric oxide, and vasopressin playing a role. ${ }^{64} \mathrm{~A}$ study by Sagoo et al ${ }^{63}$ has significantly increased our understanding 
of this disease. They studied the MRI of 12 human subjects breathing a hypoxic oxygen mixture over a period of 22 hours, simulating the conditions inducing HACE. The first observation was an increase in arterial inflow, preceding the development of cerebral edema. The authors also found a reduction in venous outflow, likely due to compression of small intracerebral venules and veins as edema develops, contributing to and exacerbating the edema. HACE is a potentially fatal, severe consequence of high altitude, with a unique pathophysiology.

\section{Conclusion}

Cerebral edema is the endpoint of a vast number of brain pathologies and causes secondary neurological damage, which is potentially fatal. Cytotoxic and vasogenic mechanisms synergistically produce the clinical picture of cerebral edema associated with the various etiologies. In-depth understanding of pathophysiology of cerebral edema remains the cornerstone for managing such cases and this knowledge is also crucial to develop future diagnostic as well as therapeutic modalities for patients with cerebral edema and related disorders.

\section{Conflict of Interest}

None declared.

\section{References}

1 Thrane AS, Rangroo Thrane V, Nedergaard M. Drowning stars: reassessing the role of astrocytes in brain edema. Trends Neurosci 2014;37(11):620-628

2 Nakada T, Kwee IL. Fluid dynamics inside the brain barrier: current concept of interstitial flow, glymphatic flow, and cerebrospinal fluid circulation in the brain. Neuroscientist 2019;25(2):155-166

3 Pillinger NL, Kam P. Endothelial glycocalyx: basic science and clinical implications. Anaesth Intensive Care 2017;45(3):295-307

4 Woodcock TE, Woodcock TM. Revised Starling equation and the glycocalyx model of transvascular fluid exchange: an improved paradigm for prescribing intravenous fluid therapy. Br J Anaesth 2012;108(3):384-394

5 Ando Y, Okada H, Takemura G, et al. Brain-specific ultrastructure of capillary endothelial glycocalyx and its possible contribution for blood brain barrier. Sci Rep 2018;8(1):17523

6 Zhu J, Li X, Yin J, Hu Y, Gu Y, Pan S. Glycocalyx degradation leads to blood-brain barrier dysfunction and brain edema after asphyxia cardiac arrest in rats. J Cereb Blood Flow Metab 2018;38(11):1979-1992

7 Keep RF, Andjelkovic AV, Xiang J, et al. Brain endothelial cell junctions after cerebral hemorrhage: Changes, mechanisms and therapeutic targets. J Cereb Blood Flow Metab 2018;38(8):1255-1275

8 NakadaT,KweeIL,IgarashiH,SuzukiY.Aquaporin-4functionality and Virchow-Robin space water dynamics: physiological model for neurovascular coupling and glymphatic flow. Int J Mol Sci 2017;18(8):1798

9 Monro A, Observations on the Structure and Function of the Nervous System. Edinburgh: Creech \& Johnson; 1823

10 Morris AWJ, Sharp MM, Albargothy NJ, et al. Vascular basement membranes as pathways for the passage of fluid into and out of the brain. Acta Neuropathol 2016;131(5):725-736
11 Rasmussen MK, Mestre H, Nedergaard M. The glymphatic pathway in neurological disorders. Lancet Neurol 2018;17(11):1016-1024

12 Tang G, Yang GY. Aquaporin-4: a potential therapeutic target for cerebral edema. Int J Mol Sci 2016;17(10):E1413

13 Fukuda AM, Badaut J. Aquaporin 4: a player in cerebral edema and neuroinflammation. J Neuroinflammation 2012;9:279

14 Stokum JA, Gerzanich V, Simard JM. Molecular pathophysiology of cerebral edema. J Cereb Blood Flow Metab 2016;36(3):513-538

15 Mahajan S, Bhagat H. Cerebral oedema: pathophysiological mechanisms and experimental therapies. J Neuroanaesth Crit Care 2016;3:22-28

16 Clément T, Rodriguez-Grande B, Badaut J. Aquaporins in brain edema. J Neurosci Res 2020;98(1):9-18

17 Werner C, Engelhard K. Pathophysiology of traumatic brain injury. Br J Anaesth 2007;99(1):4-9

18 Young W, Rappaport ZH, Chalif DJ, Flamm ES. Regional brain sodium, potassium, and water changes in the rat middle cerebral artery occlusion model of ischemia. Stroke 1987;18(4):751-759

19 Michinaga S, Koyama Y. Pathogenesis of brain edema and investigation into anti-edema drugs. Int $\mathrm{J}$ Mol Sci 2015;16(5):9949-9975

20 Hsu Y, Tran M, Linninger AA. Dynamic regulation of aquaporin- 4 water channels in neurological disorders. Croat Med J 2015;56(5):401-421

21 Manley GT, Fujimura M, Ma T, et al. Aquaporin-4 deletion in mice reduces brain edema after acute water intoxication and ischemic stroke. Nat Med 2000;6(2):159-163

22 Woo SK, Kwon MS, Ivanov A, Gerzanich V, Simard JM. The sulfonylurea receptor 1 (Sur1)-transient receptor potential melastatin $4(\mathrm{Trpm} 4)$ channel. J Biol Chem 2013;288(5):3655-3667

23 Simard JM, Woo SK, Gerzanich V. Transient receptor potential melastatin 4 and cell death. Pflugers Arch 2012;464(6):573-582

24 SimardJM,WooSK,SchwartzbauerGT,GerzanichV.Sulfonylurea receptor 1 in central nervous system injury: a focused review. J Cereb Blood Flow Metab 2012;32(9):1699-1717

25 Stokum JA, Kwon MS, Woo SK, et al. SUR1-TRPM4 and AQP4 form a heteromultimeric complex that amplifies ion/water osmotic coupling and drives astrocyte swelling. Glia 2018;66(1):108-125

26 Mehta RI, Tosun C, Ivanova S, et al. Sur1-Trpm4 cation channel expression in human cerebral infarcts. J Neuropathol Exp Neurol 2015;74(8):835-849

27 Ayata C, Ropper AH. Ischaemic brain oedema. J Clin Neurosci 2002;9(2):113-124

28 Kuroiwa T, Miyasaka N, Fengyo Z, et al. Experimental ischemic brain edema: morphological and magnetic resonance imaging findings. Neurosurg Focus 2007;22(5):E11

29 Jha RM, Kochanek PM, Simard JM. Pathophysiology and treatment of cerebral edema in traumatic brain injury. Neuropharmacology 2019;145(Pt B) :230-246

30 Donkin JJ, Vink R. Mechanisms of cerebral edema in traumatic brain injury: therapeutic developments. Curr Opin Neurol 2010;23(3):293-299

31 Winkler EA, Minter D, Yue JK, Manley GT. Cerebral edema in traumatic brain injury pathophysiology and prospective therapeutic targets. Neurosurg Clin N Am 2016;27(4):473-488

32 Sorby-Adams AJ, Marcoionni AM, Dempsey ER, Woenig JA, Turner RJ. The role of neurogenic inflammation in blood-brain barrier disruption and development of cerebral oedema following acute central nervous system (CNS) injury. Int J Mol Sci 2017;18(8):1788

33 Stocchetti N, Maas AI. Traumatic intracranial hypertension. N Engl J Med 2014;370(22):2121-2130 
34 Gorse KM, Lantzy MK, Lee ED, Lafrenaye AD. Transient receptor potential melastatin 4 induces astrocyte swelling but not death after diffuse traumatic brain injury. J Neurotrauma 2018;35(14):1694-1704

35 Volbers B, Giede-Jeppe A, Gerner ST, et al. Peak perihemorrhagic edema correlates with functional outcome in intracerebral hemorrhage. Neurology 2018;90(12):e1005-e1012

36 Ironside $\mathrm{N}$, Chen CJ, Ding D. Mayer SA, Connolly ES Jr. Perihematomal edema after spontaneous intracerebral hemorrhage. Stroke 2019;50(6):1626-1633

37 Zheng H, Chen C, Zhang J, Hu Z. Mechanism and therapy of brain edema after intracerebral hemorrhage. Cerebrovasc Dis 2016;42(3-4):155-169

38 Yehya M, Torbey MT. The role of mast cells in intracerebral hemorrhage. Neurocrit Care 2018;28(3):288-295

39 Bodmer D, Vaughan KA, Zacharia BE, Hickman ZL, Connolly ES. The molecular mechanisms that promote edema after intracerebral hemorrhage. Transl Stroke Res 2012;3, Suppl 1):52-61

40 Sehba FA, Hou J, Pluta RM, Zhang JH. The importance of early brain injury after subarachnoid hemorrhage. Prog Neurobiol 2012;97(1):14-37

41 Claassen J, Carhuapoma JR, Kreiter KT, Du EY, Connolly ES, Mayer SA. Global cerebral edema after subarachnoid hemorrhage: frequency, predictors, and impact on outcome. Stroke 2002;33(5):1225-1232

42 Weimer JM, Jones SE, Frontera JA. Acute cytotoxic and vasogenic edema after subarachnoid hemorrhage: a quantitative MRI study. Am J Neuroradiol 2017;38(5):928-934

43 Hayman EG, Wessell A, Gerzanich V, Sheth KN, Simard JM. Mechanisms of global cerebral edema formation in aneurysmal subarachnoid hemorrhage. Neurocrit Care 2017;26(2):301-310

44 Cahill J, Calvert JW, Zhang JH. Mechanisms of early brain injury after subarachnoid hemorrhage. J Cereb Blood Flow Metab 2006;26(11):1341-1353

45 Tosun C, Kurland DB, Mehta R, et al. Inhibition of the Sur1-Trpm4 channel reduces neuroinflammation and cognitive impairment in subarachnoid hemorrhage. Stroke 2013;44(12):3522-3528

46 Macdonald RL. Delayed neurological deterioration after subarachnoid haemorrhage. Nat Rev Neurol 2014;10(1):44-58

47 Østergaard L, Aamand R, Karabegovic S, et al. The role of the microcirculation in delayed cerebral ischemia and chronic degenerative changes after subarachnoid hemorrhage. J Cereb Blood Flow Metab 2013;33(12):1825-1837

48 Ahn SH, Savarraj JP, Pervez M, et al. The subarachnoid hemorrhage early brain edema score predicts delayed cerebral ischemia and clinical outcomes. Neurosurgery 2018;83(1):137-145

49 Murayi R, Chittiboina P. Glucocorticoids in the management of peritumoral brain edema: a review of molecular mechanisms. Childs Nerv Syst 2016;32(12):2293-2302
50 Heiss JD, Papavassiliou E, Merrill MJ, et al. Mechanism of dexamethasone suppression of brain tumor-associated vascular permeability in rats. Involvement of the glucocorticoid receptor and vascular permeability factor. J Clin Invest 1996;98(6):1400-1408

51 Stummer W. Mechanisms of tumor-related brain edema. Neurosurg Focus 2007;22(5):E8

52 Gerstner ER, Duda DG, di Tomaso E, et al. VEGF inhibitors in the treatment of cerebral edema in patients with brain cancer. Nat Rev Clin Oncol 2009;6(4):229-236

53 Dubois LG, Campanati L, Righy C, et al. Gliomas and the vascular fragility of the blood brain barrier. Front Cell Neurosci 2014;8:418

54 Reulen HJ, Graber S, Huber P, Ito U. Factors affecting the extension of peritumoural brain oedema. A CT-study. Acta Neurochir (Wien) 1988;95(1-2):19-24

55 Papadopoulos MC, Saadoun S. Key roles of aquaporins in tumor biology. Biochim Biophys Acta 2015;1848(10 Pt B) :2576-2583

56 Scott TR, Kronsten VT, Hughes RD, Shawcross DL. Pathophysiology of cerebral oedema in acute liver failure. World J Gastroenterol 2013;19(48):9240-9255

57 Paschoal FM Junior, Nogueira RC, Oliveira ML, et al. Cerebral hemodynamic and metabolic changes in fulminant hepatic failure. Arq Neuropsiquiatr 2017;75(7):470-476

58 Albrecht J, Norenberg MD. Glutamine: a Trojan horse in ammonia neurotoxicity. Hepatology 2006;44(4):788-794

59 Nguyen JH. Blood-brain barrier in acute liver failure. Neurochem Int 2012;60(7):676-683

60 Sekhon MS, Ainslie PN, Griesdale DE. Clinical pathophysiology of hypoxic ischemic brain injury after cardiac arrest: a "two-hit" model. Crit Care 2017;21(1):90

61 Harukuni I, Bhardwaj A. Mechanisms of brain injury after global cerebral ischemia. Neurol Clin 2006;24(1):1-21

62 Rhee CJ, da Costa CS, Austin T, Brady KM, Czosnyka M, Lee JK. Neonatal cerebrovascular autoregulation. Pediatr Res 2018;84(5):602-610

63 Sagoo RS, Hutchinson CE, Wright A, et al. Birmingham Medical Research and Expedition Society. Magnetic Resonance investigation into the mechanisms involved in the development of high-altitude cerebral edema. J Cereb Blood Flow Metab 2017;37(1):319-331

64 Hackett PH, Roach RC. High-altitude illness. N Engl J Med 2001;345(2):107-114

65 Hackett PH, Yarnell PR, Weiland DA, Reynard KB. Acute and evolving MRI of high-altitude cerebral edema: microbleeds, edema, and pathophysiology. AJNR Am J Neuroradiol 2019;40(3):464-469 